\title{
GCU
}

Glasgow Caledonian

University

University for the Common Good

\section{Experience sampling: assessing urban soundscapes using in-situ participatory methods}

Craig, Adam; Moore, David; Knox, Don

Published in:

Applied Acoustics

DOI:

10.1016/j.apacoust.2016.05.026

Publication date:

2017

Document Version

Author accepted manuscript

Link to publication in ResearchOnline

Citation for published version (Harvard):

Craig, A, Moore, D \& Knox, D 2017, 'Experience sampling: assessing urban soundscapes using in-situ participatory methods', Applied Acoustics, vol. 117, no. Part B, pp. 227-235.

https://doi.org/10.1016/j.apacoust.2016.05.026

\section{General rights}

Copyright and moral rights for the publications made accessible in the public portal are retained by the authors and/or other copyright owners and it is a condition of accessing publications that users recognise and abide by the legal requirements associated with these rights.

Take down policy

If you believe that this document breaches copyright please view our takedown policy at https://edshare.gcu.ac.uk/id/eprint/5179 for details of how to contact us. 


\section{Experience Sampling: Assessing Urban Soundscapes Using In-Situ Participatory Methods}

\section{Adam Craig, David Moore, Don Knox}

Glasgow Caledonian University, UK

\section{Abstract}

Think About Sound is a novel tool which shifts the traditional paradigm of environmental soundscape assessment using an experience sampling methodology. Over the last decade, smart phone ownership has increased immeasurably and this technology has been applied here in order to allow in-situ soundscape assessment as participants go about their daily routine. Crowdsourcing data in this way has enormous potential to create rich and diverse data sets, where both qualitative and quantitative descriptions of environmental surroundings can be gathered in a flexible and noninvasive way. The application allows the collection and assessment of environmental soundscapes using the provided set of response questions and exploiting the native audio recording application on a GPS-enabled smart phone.

This paper outlines the methodological approach used for the study and the technology employed by describing the submission procedure and the back-end processes involved in handling and collecting the data. Preliminary results from an experience sampling pilot study will also be presented and discussed as well as providing insight for future uses of such technology. The authors also propose future work with regards to the development of the application and the inclusion of crowdsourced data within an interactive online map. The continual contribution to the map allows for an evolving understanding on the public perception of environmental soundscapes.

Keywords: Soundscapes, Experience Sampling, Perception, Noise, Mobile Application

\section{Introduction}

The term 'soundscape' is widely used to describe the sonic landscape (Schafer, 1977) and can be considered the auditory equivalent of a visual landscape. According to him, three main characteristics define a soundscape: the tonality, the signal (emerging sound sources) and the sound print. His work continued to focus on the classification of sound sources according to the criteria related to the function and the meaning of those sounds. This had led to an exciting increase in cross-disciplinary research being carried out by various branches of science that focus on the sound itself, the effect on the listener, and the factors that influence the individual experience of a soundscape. This approach acknowledges the multi-factorial nature of the experience including the characteristics of the dominant sound source, the meaning of the sound as interpreted by the listener and the context in which the sound is heard (Hall et al, 2013). Soundscapes can be perceptually interpreted differently depending on content or the level at which it is heard on a perindividual basis. For example, the sound of running water may be soothing but often high in sound pressure level (Jeon et al, 2010). Some sounds can be used effectively to mask irritating sounds such 
as individual vehicles providing positive information, a warning for example, to pedestrians about their presence. Car engines provide information to the individual on the directivity, the distance from the receptor, and in some cases the size of the vehicle (Ouis, 2001). Jennings and Cain (2013) outline four main influences on the perception of a soundscape namely demographics, activity, time, and space.

These factors all influence the perception of soundscapes but few of them are directly related to the sound itself. Jennings and Cain (2013) state that presenting these influences in this way allows for clearer identification of the influences on soundscape perception. Research has found that most, if not all of these categories have a significant effect on the interpretation of sound. There is also evidence to suggest that the perception of soundscapes can evoke emotional response in brain activity whether the sound that is being heard is pleasant or unpleasant. Listening to soundscapes evoked significant activity in a number of auditory receptors in the brain (Irwin et al, 2009). Compared with soundscapes that evoked no (neutral) emotional response, those evoking a pleasant or unpleasant emotional response engaged an additional neural circuit including the right amygdala (an area of the brain which processes memory and emotional response) (Medvedev et al, 2015). A novel finding from this research is that urban soundscapes with similar loudness can have dramatically different effects on the brain's response to the environment.

Factors such as age, family background, friendship groups, occupation, and gender have implications for the way in which sounds are interpreted (Irwin et al, 2009). Yang and Kang (2005) support this theory where they found that gender had some variances in soundscape perception. Females prefer certain sounds, such as church bells, children, music in the street, and running water. Overall however, other sounds such as traffic noise are rated equally annoying between both males and females. More significantly age plays a greater role in sound perception. The same study showed that younger people (10-17 year olds) are less tolerant of sounds relating to nature whereas older people are more likely to prefer soundscapes where natural sounds occur. In a similar study by the same authors, (Yang and Kang, 2005) cultural background and environmental experience play an important role in people's judgement of sound preference. This study also revealed that those visiting the city where the tests were being held felt that they were less aware of road traffic noise. This is most likely due to the 'tourist effect' where people are less susceptible to noise annoyance due to the lack of familiarity of surroundings and brain activity concentrating on new visual stimuli (Preis et al, 2015).

Cain et al (2008)propose that it is the contextual issues faced by the listener which will influence the perception of a given soundscape and is based on an activity-centric standpoint i.e. who you are, why and how you are listening, the time of day, and the location and type of space you are in. Figure 1 outlines their framework for considering factors that influence the perception of a soundscape. Perception of sounds can be underpinned by the activity that the individual is carrying out at that moment in time. For example, street music may be annoying if someone is trying to read a book but may be more enjoyable at other times where concentration may not be an issue. Truax (1983) outlines three states of listening:

1. Analytical listening - an active or conscious activity where the listener is 'tuned in' to whatever they are listening to 
2. Listening in readiness - an intermediate type of listening where the listener's attention is ready to receive information but attention is directed elsewhere

3. Background listening - where the listener is engaged in another activity i.e. 'tuned out' of the incoming sound

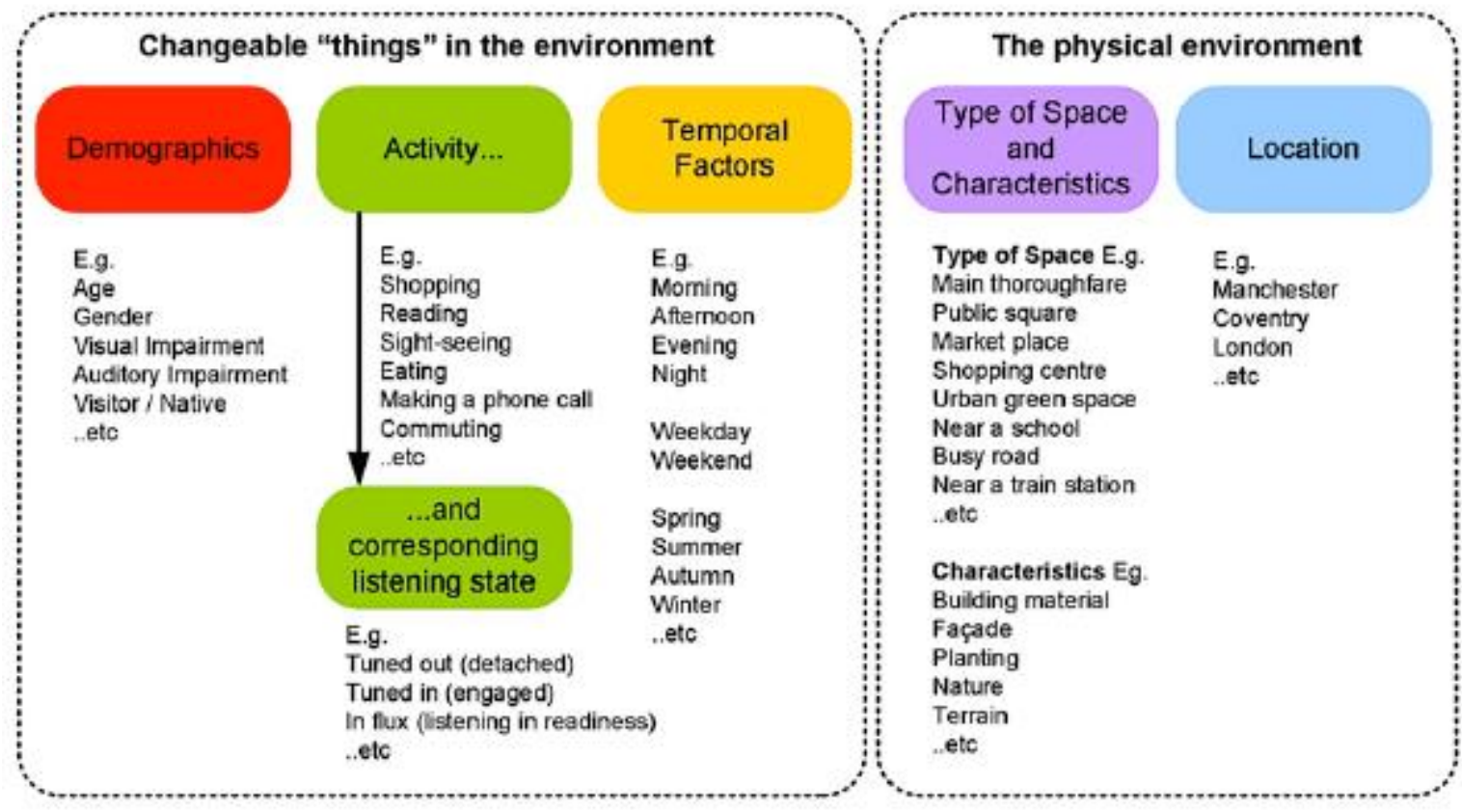

Figure 1. Cain's framework outlining the influences on the perception of soundscapes

Listening state is also important as a 'tuned in' or 'tuned out' state will affect the perception of the soundscape. In 'tuning out', listeners effectively 'hear away' from them in a manner that lets the sounds 'disappear' from their conscious sphere (Stockfelt, 1991).

It is also important to consider the effect of the temporal conditions, as the perception of a soundscape may change during the course of a day, a week, or even a year. Weekdays and weekends may produce different soundscapes in the same space (Marry, 2011). Furthermore, seasonal differences, and consequently changes in the weather may also have a significant impact. The purpose of a specific type of space i.e. whether it is in a public square, thoroughfare, busy road, shopping centre etc. will obviously have an effect on the soundscape, as will its morphology and the built landscape, so it is important to take these factors into account.

The space in which people listen to sounds can also have a resultant influence on the evaluation of those sounds (Kamenicky, 2014). It is widely regarded that tranquil areas and open public spaces can lead to comfort and can make people perceivably sense a quieter environment whereby to provide a comfortable acoustic environment the background level must be reduced in open urban spaces (Tse et al, 2012; Liu et al, 2014). Furthermore, the density of urban spaces can influence soundscape 
perception in a negative way (Yang and Kang, 2005). Results obtained in the aforementioned study revealed that participants in two different sound-walks had more positive perceptions on the environments where quiet spaces featured in the walk. The impressions were mostly influenced by the acoustic comfort and the visual imagery of the respective walks.

One important variable in soundscape perception is simply how people think about different sounds (Foale and Davies, 2012). A fundamental aspect of this is the way a listener categorises sounds (Axelsson, 2015). A range of approaches has been used to establish classifications and categorisations of both sounds and soundscapes. For example, (Maffiolo et al, 1999) asked listeners to sort urban soundscapes (based on loudness or pleasantness) and found two generic categorisation types; 'event sequences', where individual sounds can be distinguished within the soundscape and 'amorphous sequences', whereby sounds are not easily distinguishable. Other research, for example Kuwano et al (2002) has shown that a soundscape is often perceived as a collection of the individual sounds of which it is comprised; soundscape assessment is therefore influenced by the assessment of those sound types. This implies that soundscape assessment relies upon the identification of the sounds, the prominence of the sounds, and potentially the ratio of certain sound types to other sound types within the soundscape. Perhaps the most common category set that emerges from this type of research is one where listeners have classified sounds into the types 'natural', 'human' and 'mechanical' (Payne et al, 2009) Another important finding from this area of work, however, is that the category set used by listeners is contingent - it depends on several variables, especially location and soundscape.

Some isolated approaches to this matter have also been made from the fields of geography and environmental psychology. Southworth (1969), for instance, presented a pioneering study which charted the reactions of different population groups during a tour around Boston and showed that people's evaluation of a city's sound environment depends on three aspects: the information contained in the sound, the context in which it is perceived and its perceived amplitude. Anderson et al (1983) found that any appraisal of a given place depended largely on the sounds heard there. The authors used a variety of procedures such as in situ evaluation, questionnaires setting out verbal descriptions of sounds, and slides accompanied by recorded sounds. Herrington et al (1993) studied the validity of different media for representing landscapes with significant dynamic elements. More recently Viollon et al (2002) studied the influence of visual cues on auditory components in urban landscapes. Their findings were that visual conditions modify the auditory perception of subjects to a significant degree.

This research focuses on gaining a better understanding on the perception of environmental noise/soundscapes and what emotions they evoke as well as the interplay between the listener, the situation, and the noise itself. In other words, in what circumstances does the listener become aware of the soundscape which envelops them? The research community has focussed on categorising soundscapes using a number of different disciplines (ecological, sociological, and phenomenological for example). The study presented here uses a phenomenological approach and allows the participant to record their everyday responses in the place in which a sound event is heard and results in a personal measurement of presence and 'being there' (Turner et al, 2003. Phenomenology seeks to understand the variation of the participant's life-world; it attempts to explore personal experience and is concerned with an individual's personal perception or account of an object or event, as opposed to an attempt to produce an objective statement of the object or 
event itself (Smith, 2015). The study of soundscapes is about the experiences of sound, in contrast to the physical properties of sounds. It is about 'ear-mindedness' (Wagstaff, 1998) and conscious awareness of the sounds that surround us. The first-person perspective and personal experience are central, making this approach essentially phenomenological.

One unique approach to this research is that it assesses the personal responses of sound in-situ without any prior assumption to the sound being heard. Many studies focus on a retrospective account of sound where respondents are asked questions on past events resulting in a reflective, reconstruction of memory (Juslin et al, 2008). This research will use the Experience Sampling Method (ESM) approach where participants can be alerted several times a day using an electronic device and report on their surroundings/observations based on the questions set out by the researcher. It is important that the participant focuses on three main areas in assessing a soundscape:

1. Spaces that they are in everyday, such as their workplace, home and leisure environments, routes to work, and any other spaces they frequently inhabit

2. Times they are aware of any changes in those spaces, due to feeling more or less comfortable than normal, different times of the day or night, or any other changes that make them perceive the space differently

3. Any unusual or atypical places they are in, such as being on holiday or on a day trip somewhere they wouldn't normally go

This means that the research will allow for a cross-section of the participants lives and sheds light on the day-to-day sensory environments and they do not need to effectively leave the spaces in which they would normally inhabit. One advantage of ESM is that it permits the study of personal events as they unfold in their natural and spontaneous context. Another advantage is that it renders possible repeated measurements over time, so that the study obtains a better sense of whether a specific phenomenon occurs in particular recurrent patterns - for instance at specific times of the day.

\section{Experience Sampling}

The term 'experience-sampling' refers to a set of empirical methods that are designed to allow participants to document their thoughts, feelings, and actions outside the walls of a laboratory and within the context of everyday life. ESM has been used extensively in the social sciences and have also addressed research questions in medicine and communication (see Kubey et al, (1996)). The method consists of signalling participants at random times throughout a pre-determined period (such as a week) and asking them to report on various aspects of their current experience. ESM combines the approaches of field study observation with self-report diary. The main contribution of the method is being able to study experience that is normally beyond a researcher's observational scope. Sloboda et al (2001) note that the immediacy of sampling experience in real time avoids the distortions involved when asking people to report retrospectively.

ESM therefore, is ideally suited to this study as a means of collecting data about the soundscapes encountered in everyday events that is both comprehensive and contemporaneous. It provides a 
fine-grained map of the human perception of sound without relying on their reminiscences and generalisations. The primary focus of the study is to observe participants' experience of everyday soundscapes as they go about their everyday lives (more detail in the aims section).

\subsection{Types of ESM Protocol}

There are three main types of ESM protocol 1) Interval-contingent 2) signal-contingent, and 3) event-contingent. Interval-contingent protocol relies on alerts prompting the participant at fixed intervals throughout the day i.e. morning, evening, night etc. This approach is good for advance scheduling on part of the participant as they can plan their day around the alerts if they are aware of the times in which they occur. However, this can also mean that the participant has adequate emotional and cognitive preparation if fixed intervals are used. Signal-contingent protocol yields alerts at various times of the day (pseudo randomisation). This can result in a more reliable state of personal experience at that moment and not over a retrospective account where memories can become distorted. However, this protocol may be deemed to be a burden to the participants and they have to stop and respond at that moment of alert (Bolger et al, 2003). Finally, event-contingent protocol requires the participant to report their experiences soon or immediately after an event of interest and is based on the study of behaviours or events less common in life.

\subsection{Sampling Period}

There are four main considerations regarding the sampling period of an ESM study: 1) the number of observations required; 2 ) the incidence of target events and states; 3 ) the burden to participants and; 4) the anticipated compliance of the study. There are many schools of thought on the recommended length of published studies, from as few as seven per person (once daily for seven days; e.g., Conner et al, 2003; Gable et al, (2000)) to as many as 270 per person ( 3 times a day for 90 days). The average number of observations for signal-contingent studies collated by the author states that an estimates of between 56-168 (over 1-2 weeks, with 8-12 alerts per day), is sufficient. It is important to stress however, this does depend on the nature of each study and what is being measured. Similar studies on the emotional reactions to music (Juslin et al, 2008) recruited 32 participants who were alerted seven times a day over 14 days. Another study (Sloboda et al, 2001) recruited just eight participants who were sent seven alerts a day over a period of seven days.

The response rate (compliance) of observations will also be a contributory factor in the sampling period. If an expected response rate of $75 \%$ is achieved, then it may be worth extending the study or increasing the number of alerts in a 24-hour period.

\subsection{Diary Methods}

Diaries are a self-reporting method used repeatedly to examine ongoing experiences, offer the opportunity to investigate social, psychological, and physiological processes, within everyday situations. Simultaneously, they recognise the importance of the contexts in which these processes unfold (Bolger et al, 2003). Thus, diaries are designed to capture the "little experiences of everyday life that fill most of our working time and occupy the vast majority of our conscious attention" (Wheeler and Reis, 1991). They can involve intensive, repeated self-reports that aim to capture the events, reflections, moods, feelings, or interactions near the time they occur. Diary methods in psychological research build on the tradition of daily written accounts and the willingness of some 
participant to provide detail about their own experiences on a daily basis over a specific period of time (lida et al, 2012). One major advantage which has briefly being discussed in this document, is that diary reports are temporally close to the participant experience, it reduces the retrospection bias that is associated with usual survey design. They also tend to cover research questions that compatible with traditional survey design. These research questions can be broadly sorted into three major categories: 1) What are the average experiences of the individual, and do the experiences vary over time? 2) Is there systematic changes in the experience over a period of time? 3) What underlies the changes in this experience over the period of the study? (lida et al, 2008).

Although diary methods offer many benefits, especially when compared to traditional survey designs, it is important to consider what is known about their problems and limitations. One practical concern is that diary studies often require detailed training sessions to ensure that participants fully understand the protocol (Reis and Gable, 2000). Moreover, in order to obtain reliable and valid data, diary studies must achieve a level of participant commitment and dedication rarely required in other types of research studies. The burden of repeated queries and responses places substantial demands on the participant. To address this, investigators usually design diary instruments that are short and take several minutes to complete. Doing so can limit diary studies to less in-depth reporting of a phenomenon at each time of measurement.

\subsection{Technology}

More recently, there has been a shift from the traditional paradigm of using paper-based selfreporting diaries to embracing the availability of computerised technology for experience sampling studies. These include the use of palmtop computers or Personal Data Assistants (PDAs) which have been installed with specialised software that enables participants to report behaviours and experiences based on the active research. ESM software which has been used extensively in past research (Juslin et al (2008) for example) is Barrett and Barrett's (2001) Experience Sampling Program (ESP). This open source software package is used for running questionnaires, surveys or experiments on a PalmPilot or a portable computer and allows the researcher to configure the study length, signal intervals, and questions using the supplementary software on a PC. With the advent of the smartphone, technology has allowed a progressive change in the way in which ESM research is carried out. Mobile phones, in recent years, have seen an increase in processing power, embedded sensors, storage capacity and networking capabilities. They have evolved from purely as a means of communication to fully functional computers and over 5 billion people now have access to a mobile phone (Kanhere, 2011). In the United Kingdom alone, smartphone ownership had reached 34.6 million in 2014 and has been forecast to reach 43.4 million by 2017 (Statista.com, 2015\}. The availability of such devices coupled with the increase in technology has led to an exciting paradigm where large scale sensing is now possible through individual participation (participatory sensing) (Mydlarz et al, 2011; Matsuyama et al, 2014). This allows everyday members of the public to collect and share data from their surrounding environments using just their mobile device.

Participatory sensing offers a number of advantages over traditional sensor networks which entails deploying a large number of static wireless sensor devices collecting data over a pre-determined location. This can often be costly and the coverage will be limited to the locations of the sensors. With participatory sensing, costs are virtually minimal and the mobility of network carriers allows for greater coverage across much larger areas. Furthermore, the widespread availability of software 
development tools and means for deployment of software through online stores i.e. iTunes and Google Play can further enhance the experience from the public. By including people in the sensing loop, it is now possible to design applications which can inherently improve the day to day lives of individuals and communities.

\section{Method}

\subsection{PhoneGap and Cordova}

The Think About Sound application has been developed using the open-source PhoneGap platform which is able to create cross-platform apps using HTML, CSS, and JavaScript. Based on Apache Cordova, it contains the following components: 1 ) source code for a native application container for each of the supported mobile device platforms. The container renders the Cordova web application on the device; 2 ) a set of Core APIs (delivered as plugins) that provide a web application running within the container access to native device capabilities (and APIs) not normally supported by a mobile web browser; 3 ) a set of tools used to manage the process of creating application projects, managing plugin lifecycle, building (using native software development kits - SDKs) native applications, and testing applications on mobile device simulators and emulators (Wargo, 2014). PhoneGap then wraps these sections of code using the online Adobe Build Service to generate the native platform shells for store deployment (in this case iOS and Android). The application's User Interface (UI) can be developed using traditional HTML and CSS however Think About Sound's UI uses the jQuery Mobile Framework which allows a bespoke responsive design compatible across all mobile operating systems. Figure 2 summarises the application's developmental process and illustrates the flow from raw code to native packages. All data collected by the application was sent to a secure MySQL database while any audio submissions were parsed and sent directly to the server. The audio files were stored separately from the database and only referred to ensuring that the database would not become inefficient due to media files being held there.

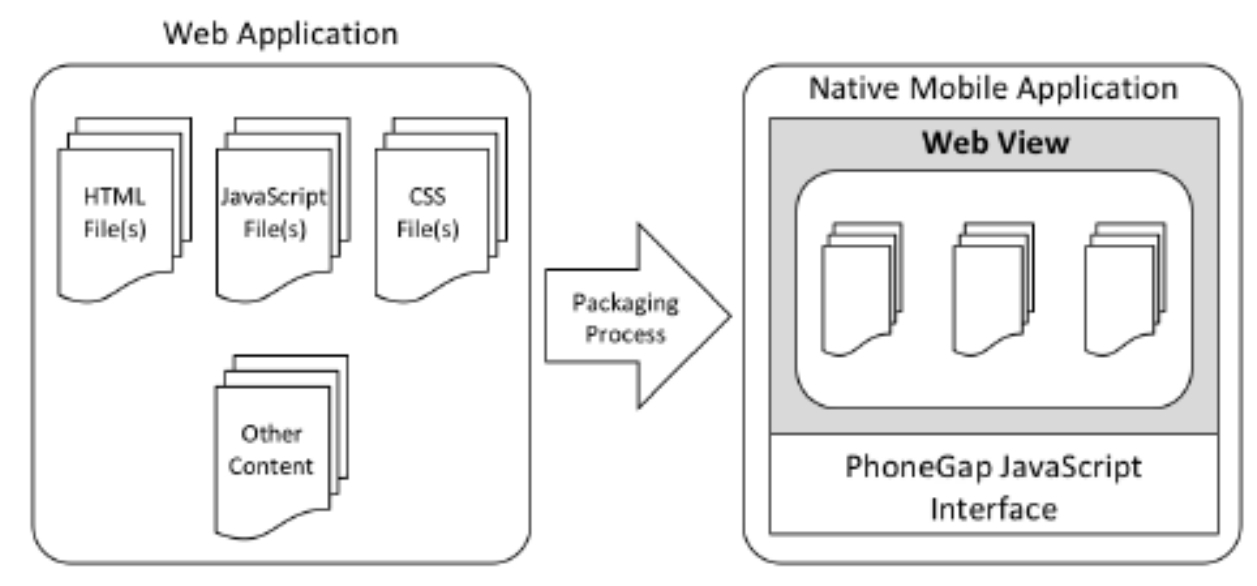

Figure 2: Developmental flow from standard web technologies to mobile application 
The Cordova platform also allows for plugins be included within the build permitting the application to access various sensors and features that would be available to native coding for each of the operating systems. These plugins offer a range of extensibility to the application and are freely available via the official Cordova plugin repository. Several of these plugins were used in the developmental stages of the Think About Sound application. Table 1 outlines the plugins and their function within the interface:

\begin{tabular}{|l|l|}
\hline Plugin Name & Purpose \\
\hline Device & $\begin{array}{l}\text { This plugin provides a description on the users phone } \\
\text { model, OS platform and version, and Unique Universal } \\
\text { Identify (UUID) of the device being used }\end{array}$ \\
\hline Splashscreen & $\begin{array}{l}\text { Allows for a splash screen to be present upon loading } \\
\text { the application }\end{array}$ \\
\hline File-Transfer & $\begin{array}{l}\text { An essential plugin required for uploading or download- } \\
\text { ing files to and from the device }\end{array}$ \\
\hline Geolocation & $\begin{array}{l}\text { Provides the devices location information such as lati- } \\
\text { tude and longitude as well as providing a timestamp and } \\
\text { IP address inferred from the connecting server }\end{array}$ \\
\hline Media-Capture & $\begin{array}{l}\text { This plugin provides access to the device's audio, video, } \\
\text { and image capturing capabilities }\end{array}$ \\
\hline
\end{tabular}

Table 1: Five of the main plugin APIs used by the Think About Sound application

\subsection{Think About Sound}

This application has been created for use with experience sampling methods whereby respondents can download the app, install, and record a response regarding the sonic soundscape they find themselves in and the personal circumstances in which they respond. The justification for using a bespoke application lies in its ability to appeal to both iOS and Android users where the majority of the market share lies across these platforms (18.3\% and $78 \%$ respectively, International Data Commission (2015)). As well as capturing audio from within the application (using the Media Capture API), users are required to provide a subjective response from their sound experience as and when a sound event occurred. Having the participant return their responses in close proximity to the sound event doesn't require reminiscing on past events resulting in more accurate responses.

The Media Capture API was programmed to respond to the user clicking on the record button within the application which would open the native recording application depending on their device. For iOS devices, this would be the Voice Recorder application capturing audio in uncompressed Waveform Audio File Format (WAV) format at a sample rate of $44.1 \mathrm{kHz}$. Android devices however, would vary greatly depending on the operating system and what applications were pre-installed by 
the smartphone's manufacturer (Android operating systems are often 'skinned' to contain custom applications and layouts between devices). This resulted in varying degrees of sound quality and file types with most devices using a compressed audio format such as Adaptive Multi-Rate (AMR), Advanced Audio Coding (AAC), and m4a audio formats with sample rates ranging from $44.1 \mathrm{kHz}$ to $8 \mathrm{kHz}$.

With increasing accuracy in Global Positioning System (GPS) data provided by mobile devices (Rao and Minakakis, 2003), the Geolocation API was used to obtain an accurate geolocation with global latitude and longitude values. This API also provides a timestamp of the moment at which the participant provides their location data. Utilising this technology has the potential to provide spatial and temporal information between the soundscape and listener.

The app itself contained five main screens accessible to the user (see Figure 3 ) the background section which provided information on the significance and rationale of the study; the instructions section that allowed participants to have an available reference guide before, and once, the study had started; the record soundscape section which allowed the user to capture the sound event using the native recording application for that phone; the submit responses section where participants answered questions on the sound event and the situation; and finally the contact us section which allowed participants (and other users) to provide feedback or questions during the study.

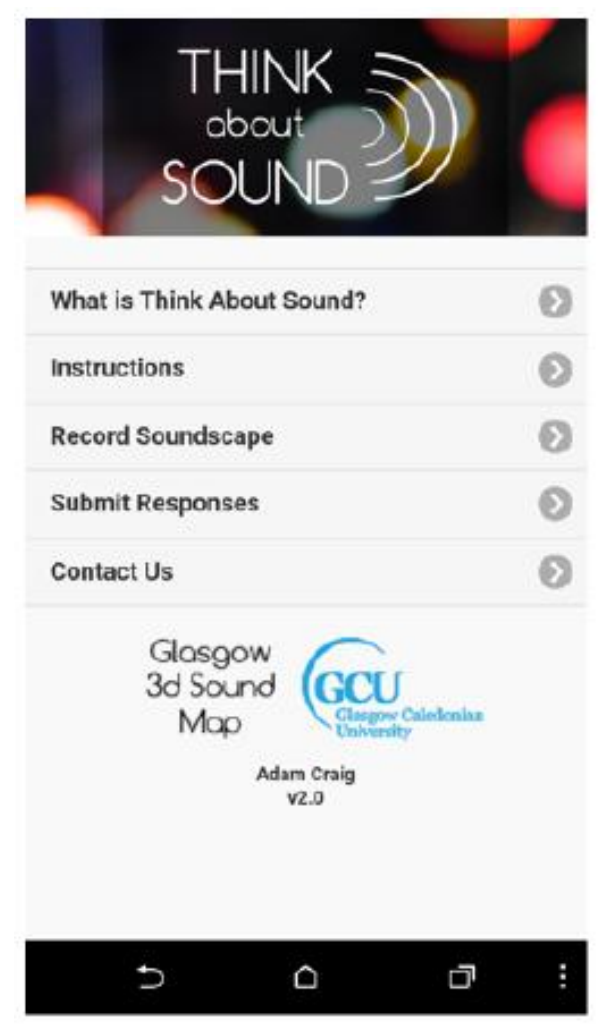

Figure 3: Application Screenshot of Main Menu 


\section{Study Implementation}

Prior to starting the data collection, comprehensive documentation was required in order to prepare the participants so that a complete understanding of what will be asked of them for the study. For the pilot study, a short demonstration of the technology and the process behind each observation was preferred to increase compliance rates throughout the duration of the study. Not only was this to meet with respondents in person and to provide instruction about the procedures but also to strengthen the 'research alliance' by providing further explanation of the study's goals and answering any questions (Hektner et al, 2007). Each participant also had to complete a short background questionnaire i.e. age, gender, contact details etc. and well as complete a consent form in order to conform to the university's ethical procedures.

Where face-to-face contact was not possible, participants were directed to the project webpage where they could access the same materials and complete the ethical consent form online. These participants were also able to contact the researchers via the same page should there be any issues prior to starting the study.

\subsection{Study Aims}

The main aim of this study was to investigate the level of activity (listening state) as it occurs naturally in everyday life with a focus on the different characteristics of the sound and how they relate to the listener, the soundscape, and the situation.

The main outcomes of the study will assess: 1) the effect of the soundscape on the listener i.e. irritation, distraction etc. 2) the situation i.e. location, alone or with another person, activity etc. and; 3) the characteristics of the soundscape itself i.e. what sounds are being heard and the prominence of those sounds at that time.

\subsection{Participants}

A total of 29 participants were recruited for the study who submitted a total number of 120 sound events over the fixed study period. They were recruited through the online website where information on the study and links to the applications on the respective stores were found. Most participants were situated within the UK and some recruited from mainland Europe and North America.

\subsection{Procedure}

Participants were asked to download the mobile application for their respective device from the relevant app store. As this study followed an event-contingent ESM protocol, participants were asked to submit a response whenever they encountered a sound which has affected them in some way whether that was a sound that was out of the ordinary or not part of their daily routine and either positive or negative. This approach enabled the assessment of rare or specialised occurrences that would not normally be captured by fixed or random interval assessments. Participants were encouraged to submit any number of sound occurrences during the 14-day study period. At each sound event, they were asked to make a short recording of the experience (around 30 seconds) to 
accompany their responses and complete the questionnaire about the situation and the sound event itself. All users were sent a daily push notification on their handsets to remind them to listen out for any sound events and the day number of the study. This notification was sent at the same time each day as not to interfere with the collection of data and was purely utilised as a tool for prompting the user during the study.

\section{Results}

A total of 120 submissions were included in the analysis of the results from the ESM study. The majority of the participants submitted an average of around 7 submissions over the study period while around $10 \%$ of the participants submitted between 8 and 14 responses, and around $20 \%$ submitting only 1 or 2 . This averaged at around 4 submissions per participants over the 14-day period. Events occurred throughout all times of the day with $70.34 \%$ the events being submitted during the day time $(7 \mathrm{am}-7 \mathrm{pm}), 23.73 \%$ occurring in the evening $(7 \mathrm{pm}-11 \mathrm{pm})$, and only $5.93 \%$ occurring during the night (11pm-7am).

The main purpose of this study was to ascertain the level of listening state as it occurs in everyday life in relation to environmental soundscapes. Can experience sampling methodology provide a substantial picture which would be consistent with retrospective data? Participants were initially asked how they were feeling before hearing the sound event which triggered a response. Figure 4 shows that the majority of participants were feeling 'calm' (40.7\%) or had a sense of 'happiness' (11\%) prior to reporting on the sound event. The figure also shows the overall change in emotions once the sound event has taken place. 'Calm' emotions dropped slightly while there was a slight rise in the level of 'anger' or 'anxiety' from the participants.

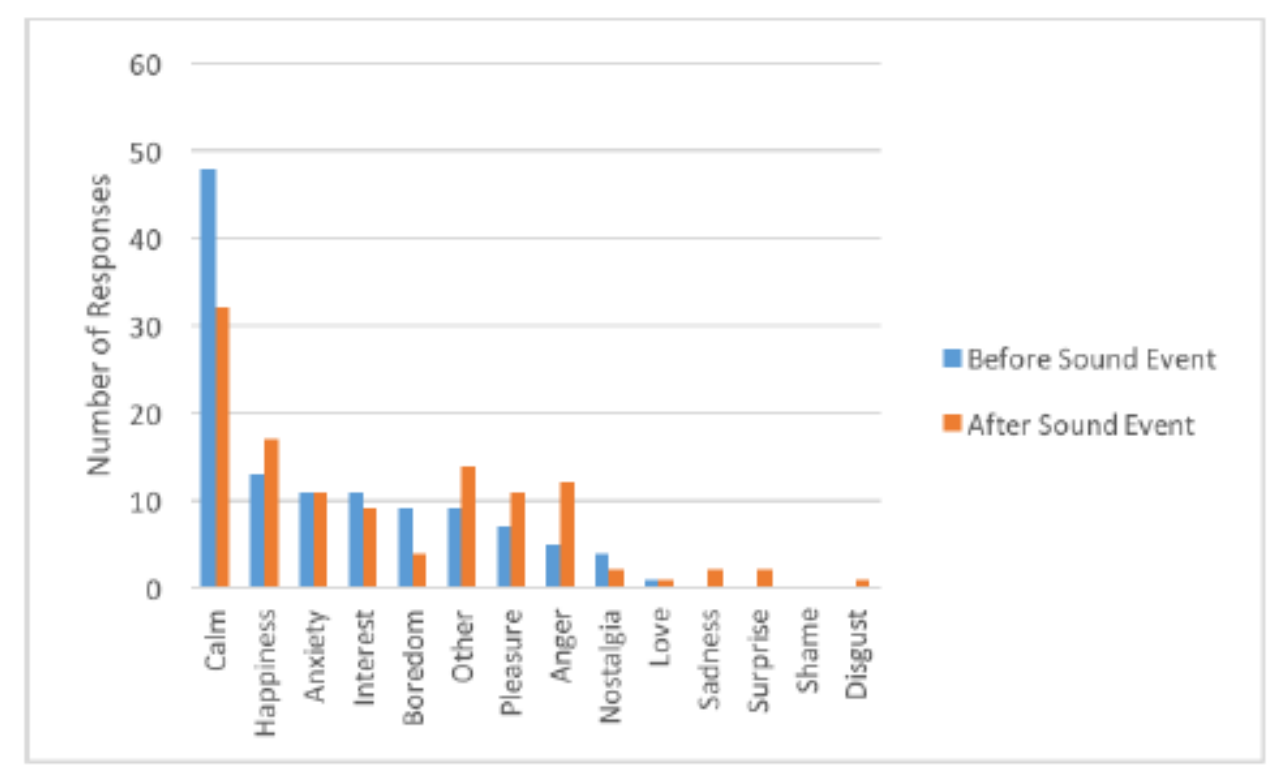

Figure 4: Overall number of submissions before and after hearing a sound event 


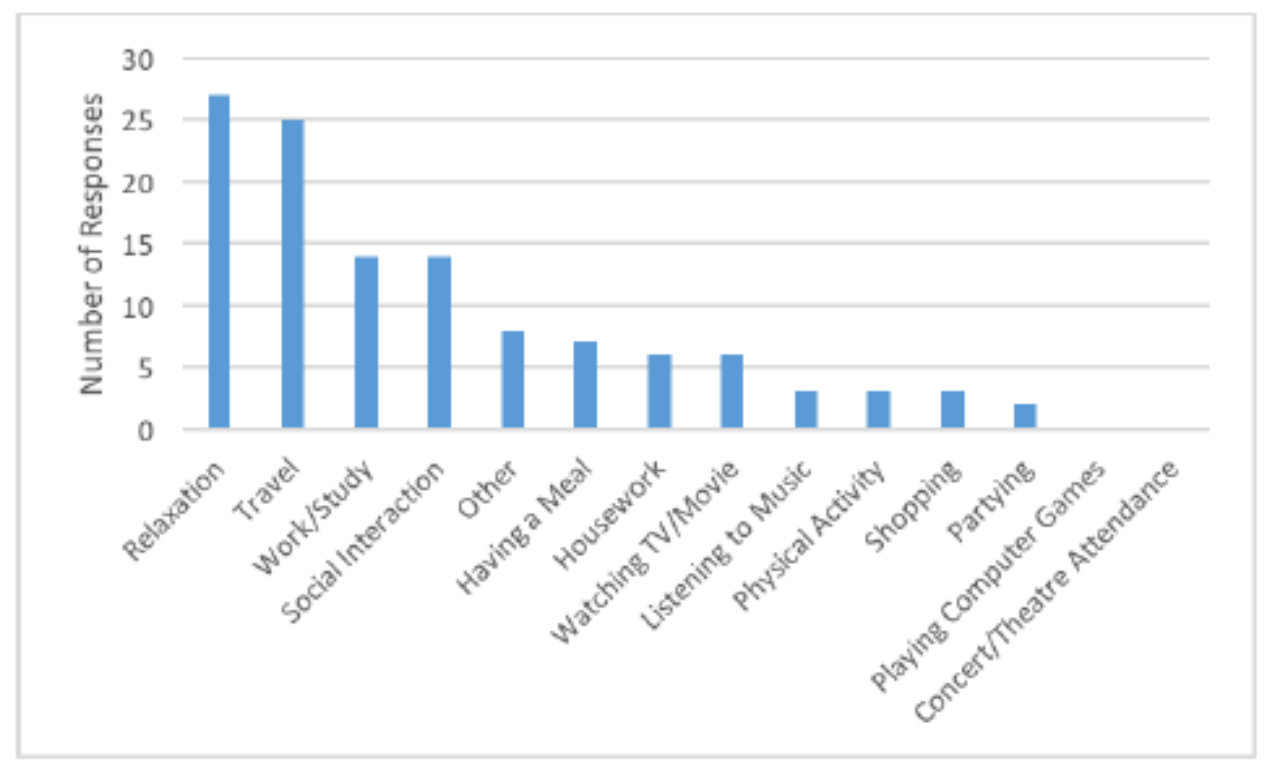

Figure 5: Reported activities of the participants when the sound event occurred

Participants' main activities were 'relaxation' and 'travel' with almost $23 \%$ and $22 \%$ of the submissions respectively reflecting this. No participants reported that they were 'Playing computer games' or at a 'Concert/Theatre performance'. Figure 5 gives an overall picture of these submissions.

Participants were mostly reported to be alone when submitting a response with $42 \%$ of all submissions. A relatively smaller portion of the submissions were with a partner or friend $(16 \%)$ or with family (15\%). People were less likely to be with several friends, be in a large crowd or with a single acquaintance or colleague. Figure 6 reveals the overall submissions for who participants were with when submitting a sound event. 


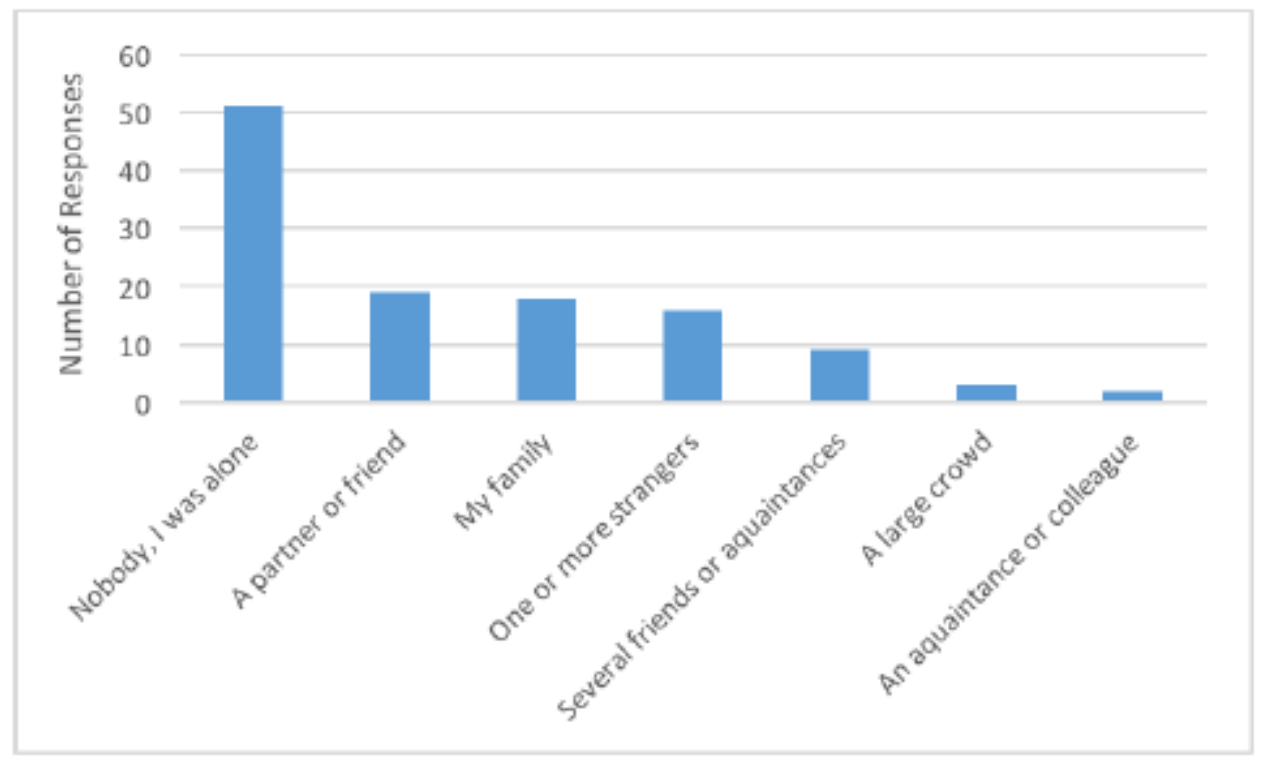

Figure 6: Categories of who participants were with when submitting a sound event

Many of the participants were alerted to a particular sound event in a positive manner whether that be 'pleasure' $(25 \%)$ or 'comfort' (22\%). 'Irritation' was a significant reason as to why participants were alerted to a sound event (24\%) and 'distraction' also had an impact with $19 \%$. A small percentage covered other reasons such as 'joy' or an awareness of being relaxed. Figure 7 provides a breakdown of the submissions per category.

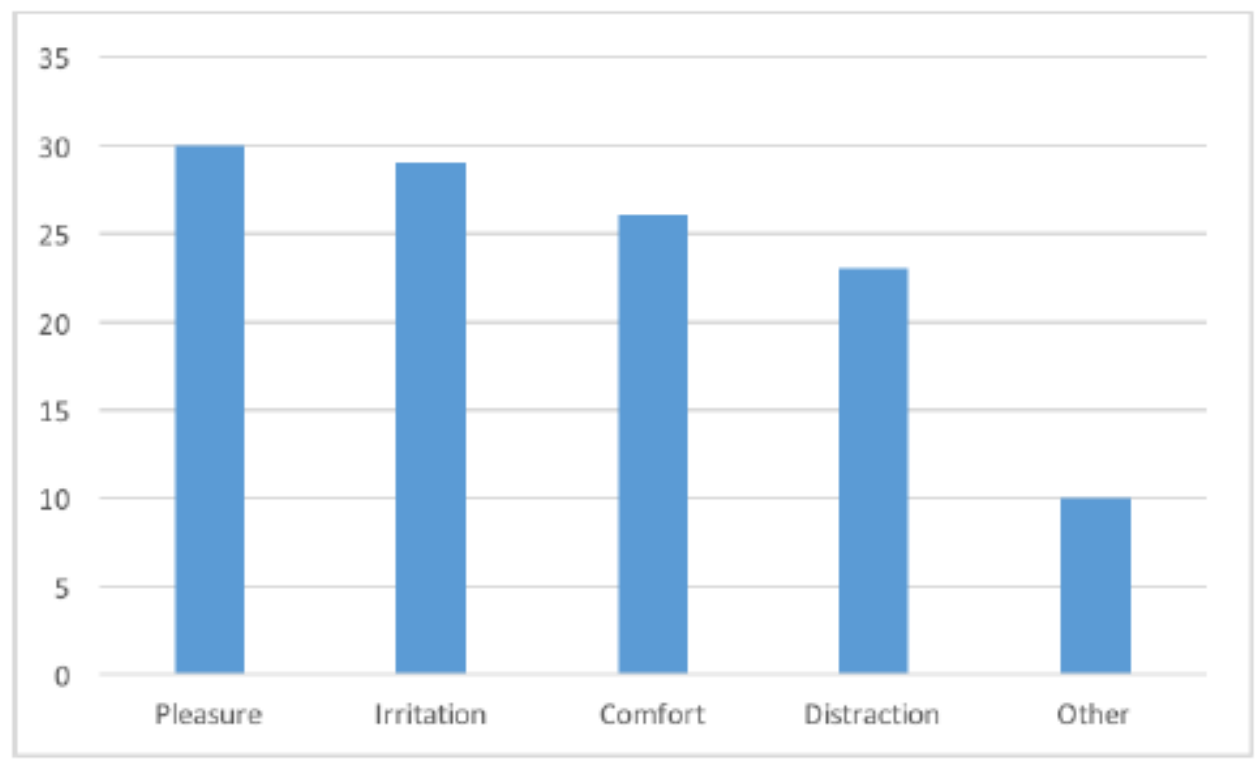

Figure 7: Categories of what alerted participants to a sound event 
Finally, respondents were ask to provide a broad category for the sound event. Many reported that 'mechanical equipment' which included transport noise, vibration from railways, and construction contributed to engaging in a sound event (29\%). 'Background noise' was also a factor (18\%) as well as 'human activity' (18\%) covering a range of sounds such as buskers, talking, music and children playing. 'Electronic' sounds were reported the least (Figure 8) perhaps due to the constant contact humans have with electronic devices etc. during their daily routine.

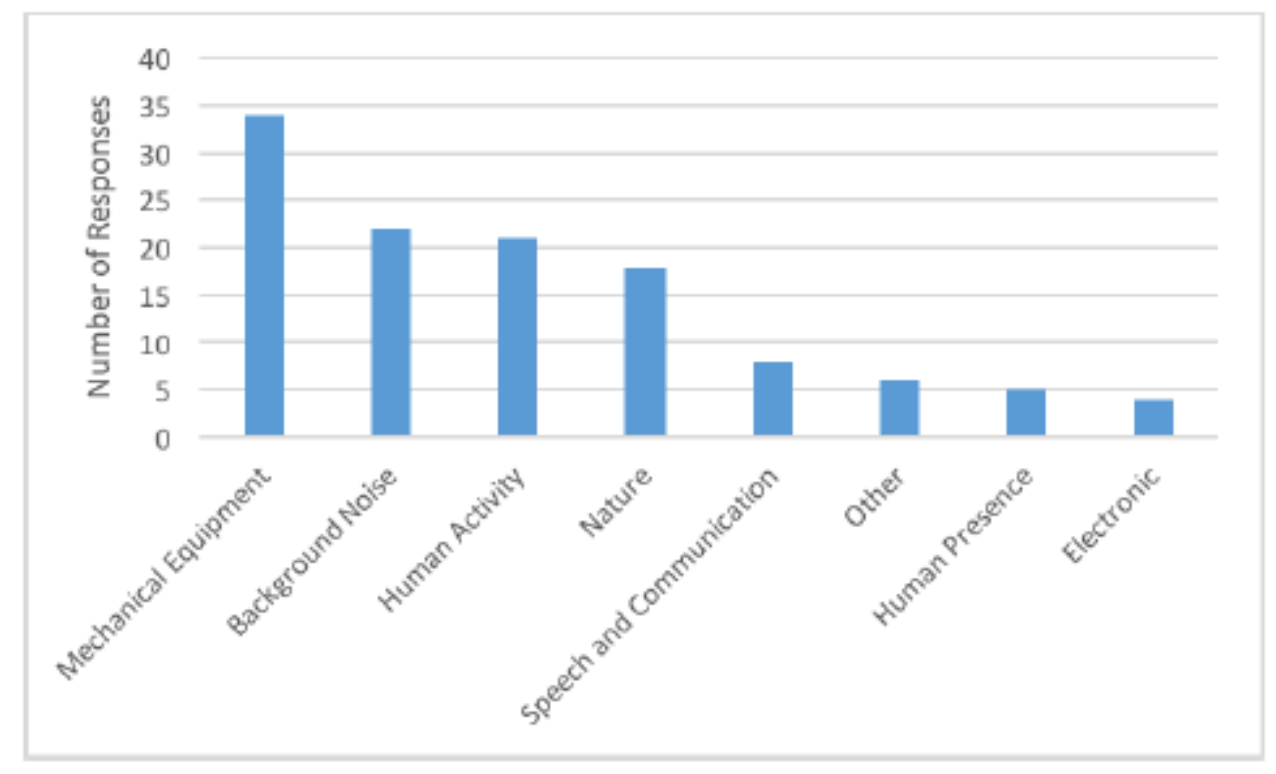

Figure 8: Categories of the sound event as reported by participants

\section{Discussion}

The development of a bespoke mobile application to aid in this ESM study was the one of the main challenges of this research. The use of the PhoneGap platform allowed cross-platform compatibility, rapid prototyping, low cost, and provided overall functionality for both the participants and the researchers. The strength of the application lies in the way in which is simple to manage and can be easily updated to reflect any changes in the question set or information required by the participants.

This initial research has been met with limited success in achieving the overall aims of the study. The level of compliance from the participants was lower than expected with only 120 submissions between 29 participants. Exit surveys revealed that some participants felt that as their daily routine was similar each day, they could not find 'new' sounds to submit to the study. Other issues raised was that the length of the study was too long for continued enthusiasm to see the study through to the end. Should the study be repeated, perhaps some sort of remuneration would encourage deeper integration into the research. Furthermore, this study employed an event-contingent protocol where participants were free to submit responses whenever they experienced a sound event which affected them in some way. Should the study have followed a more stringent process of data 
collection i.e. signal or interval contingent protocol, then more responses would likely have been achieved. Conversely, the 'personalised' approach to soundscapes would have been diluted with either of these methodologies.

Of the data that was collected, it was clear that most participants often reported more positive sounds when going about their daily routine and did so for comfort or pleasure but mechanical sound events were also prevalent throughout the study. Construction noise and road traffic were the worst offenders of negative sound events providing concurrent negative emotions i.e. irritation, distraction, or annoyance. Most participant were alone when reporting sound events and were either relaxing or travelling when providing soundscapes. This concurs with Diener and Diener (1996) who found that people in the Western world tend to experience more positive than negative emotions overall.

When reporting on the sound event, approximately $30 \%$ of the submissions had an effect on how the participant was feeling before and after the sound event. Around half of this percentage went from a positive feeling to a negative feeling and $4 \%$ moving from a negative feeling to a positive feeling. Although most submissions had no change before and after the sound event, it does suggest that there is a significant occurrence where a sound event can have an effect on how a person is feeling. Why do some sound events evoke certain changes in emotion only some of the time? It may be that some events have been encountered before or are already part of the sonic soundscape of that individual and therefore offers no real difference as they go about their daily routine. Sometimes, it may be emotional responses to environmental sounds may be prevented as something else may be happening in the foreground and those psychological resources are being used by another response.

Participants were most likely to report on a sound event from within the home or outdoors which is reasonable considering so many of the respondents were relaxing, travelling, or at work/study. A lower number of responses were given for categories such as in a cafe or bar, and in a shop or restaurant. Perhaps it is the case that those submitting responses felt that as these are mostly indoor activities, the level of outside noise would be non-existent or at the very least, almost inaudible.

Participants were also more likely to report on events when they were on their own or with a partner or friend rather than be in a large crowd or with a single acquaintance or colleague. It may well be the case that users felt that stopping to submit a sound event in this social environment would not be acceptable and therefore felt more comfortable submitting a response when it was more socially accepting in their own space with familiar people around them.

\section{Conclusion}

The advancement of mobile technology has not only encouraged and inspired in developing new means of methodological research but also increased the novel aspect to the participant. Mobile technology allows remote data collection where participants have access to question sets and other required sensors in order to contribute to future studies away from the confines of a controlled environment. This novel application in the assessment of soundscapes has proven to be valuable not 
only in this pilot study but can be adapted for any future soundscape studies. The very geolocative nature of mobile phones also allows the gathering and analysis of locative trends in soundscape appreciation and evaluation and can further provide insight into how the public perceive soundscapes based on how they were feeling or what their main activity was. This study has provided a springboard for a more in-depth study to be carried out on a much larger scale in the future.

\section{Future Work}

This study forms part of a larger research project which aims to provide the public with more meaningful sound maps based on the perception of environmental soundscapes. It addresses the limitations of current noise assessment methods (see Hepworth (2007) and Craig et al (2014)) by taking into account the relationship between the acoustic environment, human responses and the behavioural characteristics of people living within it. By using machine learning algorithms, audio files submitted as part of this ESM study and beyond will be classified from ground truth corpora in terms of arousal, valence, and annoyance as one of the overall objectives for this research cohort. Furthermore, the Think About Sound application has now been integrated into an online map where potential participants and members of the public can audition the submitted audio files and view a limited range of responses from user submissions. Further information can be found at: http://www.thinkaboutsound.co.uk.

\section{References}

Anderson, L.M., Mulligan, B.E., Goodman, L.S., and Regan, H.Z. (1983).Effects of sounds on preferences for outdoor settings. Environ. Behaviour,15 (5), pp.539-566

Axelsson, O. (2015). How to measure soundscape quality. EuroNoise 2015, Maastricht, Netherlands, 1477-1481.

Barrett, L. F., and Barrett, D.J. (2001). An introduction to computerised experience sampling in psychology. Social Science Computer Review, 19,175-185

Bolger, N., Davis, A., and Rafaeli, E. (2003). Diary methods: Capturing life as it is lived. Annual Review of Psychology 54. pp. 579-616

Cain, R., Jennings, P., Adams, M., Bruce, N., Carlyle, A., Cusack, P., Davies, W., Hume, K., and Plack, C. (2008). SOUND-SCAPE: A framework for characterising positive urban soundscapes. EuroNoise, Paris. June 29-July 4.

Conner Christensen, T., Wood, J.V., and Feldman Barrett, L. (2003). Remembering everyday events through the prism of self-esteem. Personality and Social Psychology Bulletin. Volume 29(1), pp. 5162.

Conner Christensen, T., Feldman Barrett, L., Bliss-Moreau, E., Lebo, K., and Kaschub, C. (2003). A Practical Guide to Experience Sampling Procedures. Journal of Happiness Studies 4: 53-78. 
Craig, A., Knox, D., and Moore, D. (2014). An Investigation Into The Acoustic Accuracy of Noise Maps: A Glasgow City Case Study. Proceedings of the Institute of Acoustics, Vol. 36. Pt.3.

Diener, E., and Diener, C. (1996). Most people are happy. Psychological Science, 7, 181-185.

Fastl, H. (2006). Psychoacoustic basis of sound quality evaluation and sound engineering. In The Thirteenth International Congress on Sound and Vibration. Vienna.

Foale, K., and Davies, W. (2012). A listener-centred approach to soundscape evaluation. Acoustics 2012, University of Salford, Acoustics Research Centre, Salford, UK.

Gable, S.L., Ries, H.T., and Elliot, A.J. (2000). Behavioral activation and inhibition in everyday life. Journal of Personality and Social Psychology. 78 (6), pp. 1135-1149

Hall. D., Irwin, A., Edmondson-Jones, M., Phillips, S., and Poxon, J. (2013). An exploratory evaluation of perceptual, psychoacoustic, and acoustical properties of urban soundscapes. Applied Acoustics $74,248-254$.

Hektner, J.M., Schmidt, J.A., and Csikszentmihalyi, M. (2007). Experience Sampling Method: Measuring the Quality of Everyday Life. Sage Publications Inc., California.

Hepworth, P. (2007) An overview of the impact of source data accuracy variations on the overall accuracy of noise maps. 14th International Conference on Sound and Vibration, Cairns, Australia, 15.

Herrington, S., Daniel, T.C., and Brown, T.C. (1993). Is motion more important than it sounds? The medium of presentation in environment research. Environ. Psychology 13, 283-291

lida, M., Shrout, P.E., Laurenceau, J.P., and Bolger, N. (2012). Using Diary Methods in Psychological Research. Handbook of Research Methods in Psychology: Vol. 1. Foundations, Planning, Measures, and Psychometrics.

lida, M., Seiman, G., Shrout, P.E., Fujita, K., and Bolger, N., (2008). Modeling support provision in intimate relationships. Journal of Personality and Social Psychology, 84, 460-478.

International Data Commission (2015). Smartphone OS Market Share, Q1 2015. Retrieved From: http://www.idc.com/prodserv/smartphone-osmarket- share.jsp

Irwin, A., Hall, D. A., Peters, A., and Plack, C. J. (2009). Listening to urban soundscapes: Physiological validity of perceptual dimensions. Psychophysiology, 48(2011), 258-268.

Jennings, P., and Cain, R. (2013). A framework for improving urban soundscapes. Applied Acoustics, 74(2), 293-299.

Jeon, J.Y., Lee, P.J., You, J., and Kang, J. (2010). Perceptual assessment of quality of urban soundscapes with combined noise sources and water sounds. In J. Acoust. Soc. Am., 127(3), 13571366.

Juslin, P.N., Liljestom, S., Vastfall, D., Barradas, G., and Silva, A. (2008). An Experience Sampling Study of Emotional Reactions to Music: Listener, Music, and Situation. Emotion, Vol. 8, No. 5, 668-683

Kang, J., and Zhang, M. (2010). Semantic differential analysis of the soundscape in urban open public spaces. Building and Environment, 45(1), 150-157.

Kamenicky, M. (2014). Analysis of soundscape of selected urban public places and its impact on their assessment by users. INTER-NOISE 2014, Melbourne, Australia, 1-8. 
Kanhere, S. S. (2011). Participatory sensing: Crowdsourcing data from mobile smartphones in urban spaces. In Mobile Data Management (MDM), 2011 12th IEEE International Conference on (Vol. 2, pp. $3-6)$.

Kubey, R., Larson, R., and Csikszentmihalyi, M. (1996). Experience Sampling Method Applications to Communication Research Questions. Journal of Communication, 46 (2), 99-120

Kuwano, S., Kato, N.S.T., and Hellbrueck, J. (2002). Memory of the loudness of sounds and its relation to overall impression. In: Forum Acusticum, Seville, Spain.

Liu, J., Kang, J., Behm, H., and Luo, T. (2014). Effects of landscape on soundscape perception: Soundwalks in city parks. Landscape and Urban Planning, 123, 30-40.

Maffolo, A.V., Castellengo, M., and Dubois, D. (1999). Qualitative judgements of urban soundscapes. In: INTER-NOISE 99, Fort Lauderdale, Florida, USA.

Marry, S. (2011). Assessment of Urban Soundscapes. Org. Sound, 16(3), 245-255.

Matsuyama, M., Nisimura, R., Kawahara, H., Yamada, J., and Irino, T. (2014). Development of a Mobile Application for Crowdsourcing the Data Collection of Environmental Sounds. HIMI, Part 1, LNCS 8521, 514-524.

Medvedev, O., Shepherd, D., and Hautus, M.J. (2015). The restorative potential of soundscapes: A physiological investigation. In Applied Acoustics, 96, 20-26.

Mydlarz, C., Drumm, I., and Cox, T. (2011). Application of novel techniques for the investigation of human relationships with soundscapes. INTERNOISE and NOISE-CON Congress and Conference Proceedings 2011(7), Institute of Noise Control Engineering, 738-744.

Ouis, D. (2001). Annoyance From Road Traffic Noise: A Review. Journal of Environmental Psychology, 21(1), 101-120.

Payne, S.R., Davies, W.J., and Adams, M.D. (2009). Research into the practical and policy applications of soundscape concepts and techniques in Urban Areas. Department for Environment, Food and Rural Affairs: London.

Preis, A., Kocinski, J., Hafke-Dys, H., and Wrzosek, M. (2015) Audio-visual interactions in environment assessment. Sci. Total. Environ., 523, 191-200.

Rao, B., and Minakakis, L. (2003). Evolution of Mobile Location-Based Services. Communications of the ACM, Vol. 46, Dec. 2003, p. 61.

Reis, H.T., and Gable, S.L., (2000). Event-sampling and other methods for studying everyday experience. In Handbook of Research Methods in Social and Personality Psychology. pp. 190-222

Schafer, R.M. (1977). The Tuning of the World. Knopf, New York.

Sloboda, J.A., O'Neill, S.A., and Ivaldi, A. (2001). Functions of music in everyday life: an exploratory study using the Experience Sampling Method. Musicae Scientiae, 5 (1), 9-32.

Smith, J.A. (2015). Qualitative psychology: a practical guide to research methods. 3rd edition, Sage, p26.

Southworth, (1969). The sonic environment of the cities Environment and behavior I, 49-70 
Statista.com (2015). Number of Smartphone Users in the United Kingdom (UK) from 2011 to 2017 (in Millions). Retrieved from: http://www.statista.com/statistics/270821/smartphone-user-intheunited-kingdom-uk/

Stockfelt, T. (1991). Sound as an existential necessity. Journal of Sound and Vibration, 151:3, pp. 367370.

Truax, B., (1983). Acoustic Communication, Ablex Publishing Corporation, Norwood NJ.

Tse, M.S., Chau, C.K., Choy, Y.S., Tsui, W.K., Chan, C.N., and Tang, S.K. (2012) Perception of urban park soundscape. J. Acoust. Soc. Am., 131(4), 2762-2771.

Turner, P., McGregor, I., Turner, S., and Carroll, F. (2003). Evaluating soundscapes as a means of creating a sense of place. Proceedings of the 2003 International Conference on Auditory Display, Boston, MA, USA. 149-151.

Viollon, S., Lavandier, C., and Drake C. (2002). Influence of visual setting on sound ratings in an urban environment. Applied Acoustics, 63, 493-511.

Wagstaff. G. (1998). Utopianism: from Cage to Acoustic Ecology. Hey Listen! Stockholm, Sweden, The Royal Swedish Academy of Music, 23-29.

Wargo, J. M. (2014). Apache Cordova API Cookbook. Addison Wesley. $1^{\text {st }}$ Edition. pp 1-24

Wheeler, L., and Reis, H.T. (1991). Self-recording of everyday life events: origins, types, and uses. J. Personal. 59:339-54.

Yang, W., and Kang, J. (2005). Acoustic comfort evaluation in urban open public spaces. Applied Acoustics, 66(2), 211-229.

Yang, W., and Kang, J. (2005). Soundscape and Sound Preferences in Urban Squares: A Case Study in Sheffield. Journal of Urban Design, 10(1), 61-80. 